\title{
PROPER MOTIONS WITH RESPECT TO GALAXIES
}

(Invited Paper)

\author{
A. N. DEUTSCH \\ Pulkovo Observatory, U.S.S.R. \\ and \\ A. R. KLEMOLA \\ Lick Observatory, Santa Cruz, Calif., U.S.A.
}

\begin{abstract}
At Lick the second phase of the proper motion program is in progress. In addition to generally selected stars, as was done for the first phase, so far over 30000 stars of special types of astro-physical interest and about 29000 AGK 3 stars have been selected for measurement.

In accordance with the Pulkovo program, second-epoch photography with galaxies is being continued at Pulkovo, Moscow and Tashkent, and proper motions with reference to galaxies are derived.

Analyses of proper motions at Pulkovo and Lick show agreement in some instances and disagreement in others. The same applies to comparisons with fundamental catalogues. The analyses suffer to some extent from absence of proper motions in the zone of avoidance and in the southern part of the sky.

In the southern hemisphere, first-epoch photography of 164 fields with galaxies has been completed using the Maksutov double-meniscus telescope at Cerro El Roble in Chile, and a complete coverage of the sky has been started with the same telescope; this work is being done jointly by the Soviet and Chilean astronomers. On the Yale-Columbia southern program, the first-epoch photography is nearly completed with the double astrograph at Leoncito in Argentina. There are plans at Lohrmann Institute, Dresden, to take photographs with the 2-m Schmidt telescope at Tautenburg, thus providing first-epoch plates for proper motions with reference to galaxies.
\end{abstract}

\section{Introductory Note}

One of the authors (A.N.D.) was not able to attend this symposium and his contribution communicated by correspondence has been incorporated in this report by the other author (A.R.K.).

\section{Summary of Proper Motion Programs}

The nature and present status of the various programs for the measurement of stellar proper motions with respect to galaxies is given in Table I. These comments depend in part on a review article by Vasilevskis (1973).

In the table are given the main elements of the Lick and Yale-Columbia programs based on $51-\mathrm{cm}$ aperture astrographs, as well as the original program of the Pulkovo Obșervatory using various $33-\mathrm{cm}$ Normal Astrographs. A recent addition is the 70-cm double meniscus Maksutov telescope in Chile. And finally there is the declared intention of H.-U. Sandig at Dresden to use the 2-m Universal Reflecting Telescope of the Karl-Schwarzchild Observatory in Tautenburg for the measurement of proper motions in selected fields. Descriptions of each of the programs is given in the following sections except for the Yale-Columbia program which is described by $\mathrm{A}$. Wesselink elsewhere at this symposium. 
TA B LE I

Programs for proper motions

\begin{tabular}{llllll}
\hline Program & Lick & Yale-Columbia & Pulkovo I & Pulkovo II & Lohrmann \\
\hline Location & Mt. Hamilton & Leoncito & (various) & C. El Roble & Tautenburg \\
Latitude & $+37^{\circ}$ & $-32^{\circ}$ & (various) & $-32^{\circ}$ & $+51^{\circ}$ \\
Telescope & $51-\mathrm{cm} \mathrm{DA}$ & $51-\mathrm{cm} \mathrm{DA}$ & $33-\mathrm{cm} \mathrm{NA}$ & $70-\mathrm{cm} \mathrm{DMM}$ & $134-\mathrm{cm}$ \\
$f /$ & 7.3 & 7.3 & 10.0 & 2.9 & 3.0 \\
Scale $(" / \mathrm{mm})$ & 55.1 & 55.1 & 60.0 & 103.1 & 53.2 \\
Field $(\mathrm{deg})$ & 6.3 & 6.3 & 2.0 & 5. & 3.4 \\
Mag. Limit & $19 \mathrm{~B}, 18 \mathrm{~V}$ & $19 \mathrm{~B}, 18 \mathrm{~V}$ & 14 & 19 & $20 \mathrm{~B}$ \\
Sky Region & $+90^{\circ}$ to $-23^{\circ}$ & $-90^{\circ}$ to $-22^{\circ}$ & galaxies & S. sky/galaxies & $\mathrm{N}$. sky zones \\
No. Fields & 1246 & 598 & $300:$ & $-/ 164$ & 1400 \\
\hline
\end{tabular}

a Telescopes are denoted $\mathrm{DA}=$ Double Astrograph, NA $=$ Normal Astrograph, and DMM $=$ DoubleMeniscus Maksutov. Aperture of the Schmidt corrector given for the 2-m Tautenburg telescope.

\subsection{LICK PROGRAM}

The distribution of fields and status of observations is shown in Figure 1. The total number of fields is 1246 and 1390 to southern declination limits of $-23^{\circ}$ and $-33^{\circ}$, respectively. Although the original program as proposed by W. H. Wright extends to a limit of $-23^{\circ}$, an examination of supplementary plates taken by Shane and Wirtanen reveals that plates with centers at $-25^{\circ}$ are of acceptable quality for measurement as well as the central areas of plates with centers at $-30^{\circ}$. It is estimated that the number of fields with a sufficient number of galaxies to obtain plate constants amounts to 926 and 1031 to $-23^{\circ}$ and $-33^{\circ}$, respectively. Therefore the fraction of fields lying outside the zone of avoidance is $74 \%$. As of 1973 June the total number of fields with acceptable second-epoch plates came to 336. These are located mostly away from the Milky Way, as seen from Figure 1.

One of the main goals of the program is the determination of corrections to the motions in the FK4 system and the constants of precession. Since the stars of the FK4 are too bright for measurement on blue astrograph plates, it is necessary to select fainter stars from the AGK3. It should be pointed out that the yellow plates may be used at a future third epoch to measure motions of the fainter FK4 stars, say as bright as magnitude 5.0, since the growth of image size with magnitude is much smaller than for the blue plates. The selection of stars in three ranges of magnitude was made from a magnetic tape containing the AGK3. The density of selected stars on the sky from declination $+90^{\circ}$ to -2.5 is shown in Table II. The number of stars selected is 28821 from a total of 183572 in the AGK3, or $15.8 \%$. If an estimated $70 \%$ survive to the final stage of reduction, then the final number will be near 20000 , or $11.0 \%$ of the AGK3. Since this figure includes the sky within the zone of avoidance, the number lying outside and thus measured directly with respect to galaxies should be about $74 \%$ of this figure, or 15000 stars.

Catalogue stars for the magnitude range 7 to 12 for the sky southward from dec- 


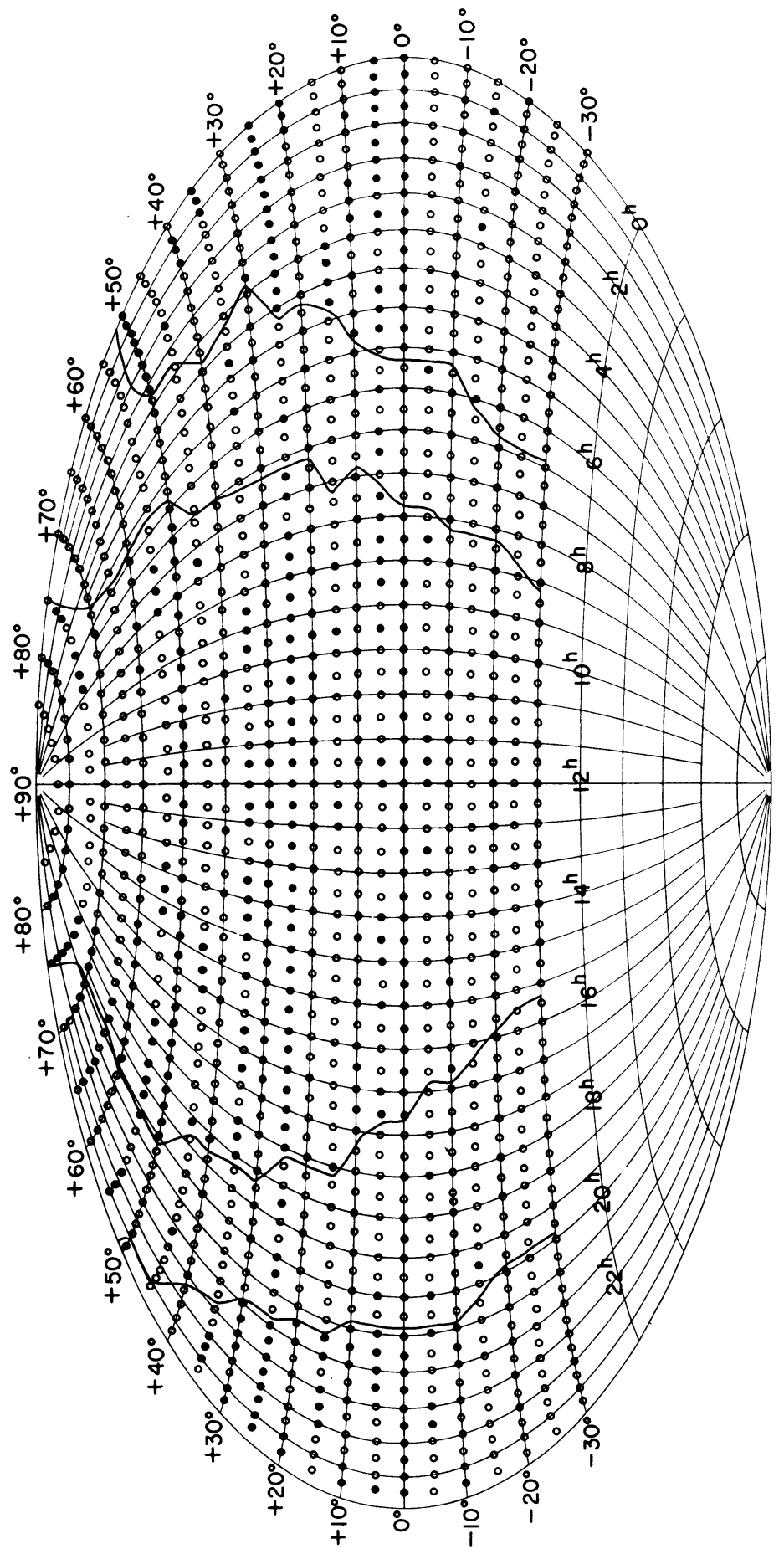

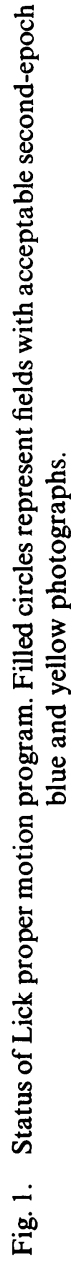


TABLE II

Number of stars per square degree selected from AGK3

\begin{tabular}{lllll}
\hline AG & Group & Bright & Medium & Faint \\
\cline { 2 - 5 } Zone & Mag. & $7.0-8.5$ & $8.6-10.2$ & $10.3-12.0$ \\
\hline$+89^{\circ}$ to $+86^{\circ}$ & 0.33 & 1.0 & 1.0 \\
$+85^{\circ}$ to $-1^{\circ}$ & 0.33 & 0.5 & 0.5 \\
$-2^{\circ}$ & 0.66 & 1.0 & 1.0 \\
\hline
\end{tabular}

lination -2.5 will be taken from the Smithsonian Astrophysical Observatory Catalogue with a density the same as for the northern sky for the three magnitude groups.

To permit the transformation of iris photometer readings into magnitudes and color indices in the $B, V$ system, stars are selected from the photometric catalogue of Blanco et al. (1968). Most of these stars are brighter than mag. 13, so that only an approximate reduction will be possible for the faint program stars in most fields.

One of the important expected contributions from the program is the measurement of proper motions for various classes of stars chosen from the literature on the basis of probable astrophysical or kinematical interest. At the meeting of the IAU at Brighton in 1970 it was reported that as many of the RR Lyr stars as possible would be measured. Since then the scope of the program has been enlarged greatly. Now a selection of all available members of many classes of stars has been undertaken. The details of the selection are shown in Tables III and IV. In all there are about $\mathbf{3 0 0 0 0}$ stars of a special character. The inclusion of new stars continues as new publications are received. Duplicate entries for the same star will be sorted out as the stars are viewed in the survey machine.

In Table III an asterisk denotes an incomplete selection of a particular class of variable star. It is not intended to include objects for which the types are unknown or are constant in light. Supernovae are excluded. At least a partial selection of eclipsing systems will be made. The total selection north of declination $-33^{\circ}$ amounts to 7424 variables of the total 20448 given in the General Catalogue of Variable Stars (Kukarkin et al., 1969).

In Table IV are given other classes of stars, some of which are found in the listing of variable stars. In addition QSO's and Zwicky's compact galaxies are included in the program. These will serve as additional reference objects. Finally fainter stars appearing in the lists of the International Polar Motion Service for observation with PZT's have been selected.

By measuring motions for as many as possible of a given class of star, it will be possible to attempt finer division into subgroups for analysis of smaller variations of mean absolute magnitude. To permit maximum usefulness of the proper motion data, it would be desirable that radial velocity and photometric data be obtained for as many of these stars as possible. Later when the Yale-Columbia southern pro- 
TABLE III

Selection of variable stars

from General Catalogue of Variable Stars (1968)

\begin{tabular}{lcc}
\hline Type & GCVS (1968) & North $-33^{\circ}$ \\
\hline Cepheid & 706 & 441 \\
Irregular & 1687 & 1458 \\
Mira & 4566 & $826^{\mathrm{a}}$ \\
Semi-regular & 2221 & 1733 \\
RR Lyrae & 4433 & 2426 \\
RV Tauri & 104 & 79 \\
$\beta$ Cephei & 23 & 17 \\
$\delta$ Scuti & 17 & 17 \\
$\alpha^{2}$ CVn & 28 & 27 \\
& & - \\
All Pulsating & 13782 & 7024 \\
\hline Nova/Nova-like & 203 & 153 \\
R Cor Bor & 32 & 15 \\
Irregular & 1109 & $0^{\mathrm{a}}$ \\
UV Ceti & 28 & 24 \\
U Gem & 215 & 162 \\
Z Cam & 20 & 18 \\
Supernova & 7 & 0 \\
& - & 372 \\
All Eruptive & 1618 & 28 \\
\hline Eclipsing & 4062 & 0 \\
Unique & 35 & 0 \\
Unstudied & 803 & $0^{\mathrm{a}}$ \\
Constant & 148 & \\
All Other & 5048 & \\
\hline
\end{tabular}

a Very incomplete selection.

gram is completed, there will be on hand motions for variable and non-variable stars for the whole sky. This full sample should then permit a better determination of stellar motions and catalogue corrections than is possible today.

As in the pilot program, faint anonymous stars are selected for measurement. Experience with the pilot program showed that a wider range of magnitude was desirable. Consequently two stars per square degree are selected: one of mag. 14-15 and another of mag. 15-17 for a total of 72 per field of $6^{\circ} \times 6^{\circ}$.

The selection of galaxies remains the same as for the pilot program, namely, at least one per square degree where possible ( 72 per plate and up to 100 in rich fields).

\subsection{Pulkovo programs}

The Pulkovo proper motion programs may be discussed in three parts. First, there is the original program using various Normal Astrographs for which results of mea- 
TABLE IV

Selection of other classes of objects

\begin{tabular}{lclc}
\hline Class & Number & Class & Number \\
\hline Ap, Am & 394 & Metal-deficient & 300 \\
Barium & 150 & Miscellaneous & 1215 \\
Carbon (R, N) & 1629 & O-B & $94^{\mathrm{a}}$ \\
Compact galaxies & 1934 & Planetary nebulae & 485 \\
Common proper motion & 892 & QSO & 545 \\
Faint blue objects & 6226 & Red (giants, dwarfs) & 6850 \\
Helium & 10 & Subdwarfs & 416 \\
Horizontal-branch & 53 & White dwarfs & 900 \\
Infra-red & 285 & Wolf-Rayet & 66 \\
IPMS (PZT) & $96^{\mathrm{a}}$ & X-ray & 2 \\
\hline & Sum & & 22542 \\
\hline
\end{tabular}

${ }^{\text {a }}$ Very incomplete selection.

surements and analysis have been published for 85 fields. Second, there is the current program which is the continuation of the second-epoch photography with the Normal Astrographs. Deutsch in his report to Commission 24 reports that plates taken at Pulkovo from 1949 to 1958 serve for the first epoch and are repeated once the epoch difference reaches 20 years. Stars down to mag. 14 are measured. At Moscow and Tashkent the new plates are compared with those taken before 1941. The third part of the Pulkovo programs consists of the application of the 70-cm double-meniscus Maksutov telescope at Cerro El Roble for a southern program in two parts: (1) the recently completed first-epoch photography for 164 fields with galaxies and (2) the recently started full coverage of the southern sky with blue and yellow plates. Plates are taken with long ( $30 \mathrm{~min})$ and short (1-3 min) exposures and will serve to determine absolute proper motions of stars down to magnitude 19.

At Pulkovo (comments by A.N.D.) preliminary results of new measurements with the semi-automatic measuring machine ASCORECORD confirm the earlier conclusion that

$$
\text { p.e. }(\text { galaxy })=1.5 \text { p.e. }\left(\text { reference stars, } \bar{m}_{p g}=13.8\right)
$$

and

$$
\text { p.e. }(\text { galaxy })=\text { p.e. }(\text { AGK3 star }) \text {. }
$$

The measured 'proper motion of a galaxy' with respect to faint reference stars for one plate pair is \pm 0.008 to $0.010 \mathrm{yr}^{-1}$. It is expected that future measurements of the second-epoch Normal Astrograph plates will yield a p.e. for a galaxy or AGK3 star of \pm 0.002 to $0.003 \mathrm{yr}^{-1}$ from about 12 stars in a circle $50^{\prime}$ radius from 3 pairs of plates with an average of 3-4 galaxies per field.

The belief is further emphasized that the best method of measurement is through the use of a blink comparator. It should be faster and more precise since the measurer 
sees both plates simultaneously. However, such a device as envisioned here does not now exist.

The analysis of proper motions yields a solar apex that differs from the standard apex for both Pulkovo and Lick with the former being even further off than the latter. Proper motions obtained from early studies of Selected Areas showed the same effect but to a lesser degree. The Pulkovo mean secular parallaxes appear twice as large as in previous investigations, particularly at high galactic latitudes. The outstanding feature of the study of the differences in proper motions between Pulkovo and the AGK 3 is that the run of differences in proper motions in declination as a function of declination reaches $0.007 \mathrm{yr}^{-1}$ for declinations south of $+40^{\circ}$. This is not observed for the Lick motions.

\subsection{LOHRMANN PROGRAM}

Plans are underway by H.-U. Sandig of the Lohrmann Institute in Dresden, GDR, to use the 2-m Universal Reflecting Telescope of the Karl-Schwarzschild Observatory used in the Schmidt-system mode in a program of proper motions with respect to galaxies. The purpose of the program is the determination of errors of meridian circle catalogues after repetition of the plates in about 20 years.

Fields selected for the program will overlap center-to-center. The fields to be observed lie along four parallels of declination: $0^{\circ},+26^{\circ},+52^{\circ}$, and $+75^{\circ}$. In addition, other fields to be observed lie along hour circles in the northern hemisphere at every two hours of right ascension. In all there will be about 1400 plates of $10^{\mathrm{m}}$ exposure each reaching mag. 20. Deutsch suggests that the fields of galaxies of the Pulkovo program be observed also. This would involve two plates for each of 140 fields.

\section{Some Outstanding Problems}

Once the absolute proper motions are on hand, there still remain several outstanding problems that hinder a best possible determination of solar motion, galactic rotation, and mean absolute magnitudes.

There is a distinct need for adequate magnitude and color standards for the fainter stars, say beyond mag. 13. As the determination of these photometric quantities falls outside the scope of the observations being carried out at Lick, it is hoped that such work will be carried out at other observatories.

Another problem is the need for adequate correction for interstellar absorption and reddening. Since stars as faint as mag. 18 are observed, corrections for the more luminous stars will be needed to great distances from the Sun. Representation of the absorption and reddening by more complex methods than the commonly used cosecant or exponential formulae would seem more realistic. One approach could be the use of the detailed analysis by Fitzgerald (1968) based on stars from the photometric catalogue of Blanco et al. (1968) which is modified for rapid computer reductions.

The bridging of the zone of avoidance may be approached from several ways. As seen earlier, about $26 \%$ of the program fields at Lick have an insufficient number or 
poor distribution of galaxies to permit the determination of plate constants. However, a portion of these Milky Way fields do have several galaxies, so that zero-point corrections to arrive at absolute motions in the manner of Pulkovo may be obtainable. Alternatively, the AGK3 stars, with motions corrected by extrapolated values found at higher latitudes, may be employed in the zone of avoidance. The various methods may be tested.

The programs now in progress should eventually provide a much stronger observational basis for the system of proper motions as well as the conclusions concerning solar motion, mean parallaxes, and galactic dynamics.

\section{References}

Blanco, V. M., Demers, S., Douglass, G. G., and Fitzgerald, M. P.: 1968, Publ. U.S. Naval Obs., 2nd Ser. 21.

Fitzgerald, M. P.: 1968, Astron. J. 73, 983.

Kukarkin, B. V., Kholopov, P. N., Efremov, Yu. N., Kukarkina, N. P., Kurochkin, N. E., Medvedeva, G. I., Perova, N. B., Fedorovich, V. P., and Frolov, M. S.: 1969, General Catalogue of Variable Stars (GCVS 1968).

Vasilevskis, S.: 1973, Vistas in Astronomy 15, 145.

\section{DISCUSSION}

Bok: When may we expect to have the proper motion for the RR Lyrae variables outside the zone of avoidance, say 2000 stars? What will be your Lick magnitude limit?

Klemola: The main work is concerned with that part of the sky outside the zone of avoidance. This is about $74 \%$ of the full programme. The results should be on hand in about 4 or 5 years. A useful magnitude limit is about 17.5 .

Murray: Could you consider including some faint red stars, say 16-17 mag., in your survey? These would have to be selected photometrically; perhaps Prof. Luyten has plate material from which extreme red faint objects could be detected.

Klemola: Yes, faint red stars could be included. What star lists would you suggest?

Murray: This would need some careful planning.

Brosche: What are the measurement principles for galaxies?

Klemola: The scanner of the measuring machine performs centring on some sort of density centre.

Luyten: Since I determine relative proper motions on the Palomar plates I am very much interested in obtaining data which would give us the correction from relative to absolute motion for various magnitudes all over the sky. How soon can we expect this?

Klemola: Present efforts are concerned with zones around declinations $0^{\circ},+25^{\circ}$, and $+50^{\circ}$. These partial results should be available in a year or two. 